\title{
Comparing Performance Potentials of Classical and Intuitionistic Fuzzy Systems in Terms of Sculpting the State Space
}

\author{
Jerry M. Mendel, Life Fellow, IEEE, Imo Eyoh, Member, IEEE, and Robert John, Senior Member, \\ IEEE
}

\begin{abstract}
This paper provides new application-independent perspectives about the performance potential of an intuitionistic (I-) fuzzy system over a (classical) TSK fuzzy system. It does this by extending sculpting the state space works from a TSK fuzzy system to an I-fuzzy system. It demonstrates that, for piecewiselinear membership functions (trapezoids and triangles), an Ifuzzy system always has significantly more first-order rule partitions of the state space-the coarse sculpting of the state space-than does a TSK fuzzy system, and that some I-fuzzy systems also have more second-order rule partitions of the state space-the fine sculpting of the state space-than does a TSK fuzzy system. It is the author's conjecture that, for piecewiselinear membership functions (trapezoids and triangles): It is the always-significantly greater coarse (and possibly fine) sculpting of the state space that provides an I-fuzzy system with the potential to outperform a TSK fuzzy system; and, that a type-1 I-fuzzy system has the potential to outperform an interval type- 2 fuzzy system.
\end{abstract}

Index Terms-intuitionistic fuzzy sets, intuitionistic fuzzy systems, TSK fuzzy systems, rule partitions, sculpting the state space.

\section{INTRODUCTION}

$\mathrm{R}$ ECENTLY, Mendel [1], [2] explained the performance potential $^{1}$ of type-1 (T1), interval type-2 (IT2) and general type-2 (GT2) rule-based fuzzy systems (fuzzy systems, for short) that use a singleton fuzzifier and piecewise-linear membership functions (trapezoids and triangles) as a greater sculpting of the state space. Mendel, et al [3], extended [1] to T1 and IT2 fuzzy systems that use a non-singleton fuzzifier. This paper extends [1] to T1 intuitionistic (I-) fuzzy systems.

In a nutshell (more formal discussions are given in Section

Manuscript received on ...

J. M. Mendel is with the University of Southern California, Los Angeles, CA 90089-2564, USA, and also with the College of Artificial Intelligence, Tianjin Normal University, Tianjin 300384, China (e-mail: mendel@sipi.usc.edu).

I. Eyoh is with the Department of Computer Science, University of Uyo, Akwa Ibom State, Nigeria (e-mail: imoeyo@gmail.com).

R. John is with the Laboratory for Uncertainty in Data and Decision Making and the Automated Scheduling, Optimization and Planning Research Groups, University of Nottingham, Nottingham NG7 2RD, UK (e-mail: rij@cs.nott.ac.uk).

${ }^{1}$ As in [1]-[3], interpretability, as a performance metric, is outside of the scope of this paper.
II), a T1 intuitionistic fuzzy set (I-FS), introduced by Atanassov [4], is described by two functions, a membership function (MF) and a non-membership function (NMF), where the constraint $\mathrm{MF}+\mathrm{NMF} \leq 1$ must always be satisfied. A T1 I-fuzzy system is described by two subsets of rules, one for the MFs and the other for the NMFs; its output can be obtained in either of two ways [5], [6]: (1) the MF and NMF sub-systems are coupled by taking a linear combination of their outputs, or (2) the two subsets of rules are viewed as one larger set of rules, and then their outputs are aggregated in the usual way by means of some defuzzification method.

I-FSs have found usefulness in diverse application domains, e.g., aiding human decision-making [7]-[12], medical diagnosis [13]-[15], image segmentation [16]-[18] and time series forecasting [19]-[21]. There are many references for T1 (and even IT2) I-FSs and applications for them. We refer the readers to [22]-[24] for very comprehensive discussions as well as many references about them. Due to page limitations, we do not include any of that material herein.

It was recently shown $[5,6,29,30]$ that an I-fuzzy system can outperform a (classical) T1 fuzzy system ${ }^{2}$. We want to be able to understand and explain: Why does this occur?

Those readers who are already familiar with a T1 I-fuzzy system may answer this question by explaining that a T1 Ifuzzy system has more rules and design parameters than a T1 fuzzy system, and using both the MF and NMF permits more flexibility than only using the MF, and so these are reasons for a T1 I-fuzzy system outperforming a T1 fuzzy system.

The goal of this paper is to provide further understanding of the performance ${ }^{3}$ improvement potential of a T1 I-fuzzy system over a T1 fuzzy system, because it is only if such performance improvement potential exists should one even consider using a T1 I-fuzzy system. This goal is accomplished herein by providing new additional explanations for the improved performance in terms of sculpting the state space due to using I-FSs. The author's conjecture is that, for piecewise-linear MFs (trapezoids and triangles): It is the always significantly greater coarse (and possibly finer) sculpting of the state space accomplished by using I-FSs that

${ }^{2}$ [5] is for classification (it uses classification accuracy as the metric) whereas $[6,29,30]$ are for regression (they use RMSE as the metric).

${ }^{3}$ As used here, "performance" is application dependent (e.g., in forecasting it could be a small RMSE, in control it could be low overshoot, in classification it could be low misclassification, etc.; however, the results in this paper [1] "provide a common component to all performance analyses, after which the rest of the performance analyses is application dependent." 
provides a T1 I-fuzzy system with the potential to outperform a T1 fuzzy system.

\section{BACKGROUND}

\section{A. I-FS (and Notations)}

An I-FS $A^{*}$, proposed by Atanassov [4], is a generalization of a FS, $A$; it consists of a MF $\mu_{A^{*}}(x) \in[0,1]$ and a NMF $v_{A^{*}}(x) \in[0,1]$, where, $0 \leq \mu_{A^{*}}(x)+v_{A^{*}}(x) \leq 1$, i.e.

$$
A^{*}=\left\{\left(x, \mu_{A^{*}}(x), v_{A^{*}}(x)\right) \mid x \in X, 0 \leq \mu_{A^{*}}(x)+v_{A^{*}}(x) \leq 1\right\}
$$

Atanassov [4] also defined the hesitancy, $\pi_{A^{*}}(x)$, of whether $x$ is in $A^{*}$ or not, as:

$$
\pi_{A^{*}}(x) \equiv 1-\left[\mu_{A^{*}}(x)+v_{A^{*}}(x)\right]
$$

When $\pi_{A^{*}}(x)=0 \quad v_{A^{*}}(x)=1-\mu_{A^{*}}(x)$, so that $A^{*} \rightarrow A$.

When one creates $A^{*}$ by starting with $A$, as we shall do, then the star notation in (1) can be simplified, i.e., in this paper, we use the following notations interchangeably:

$$
\left\{\begin{array}{l}
\mu_{A^{*}}(x) \leftrightarrow \mu_{A}(x) \equiv A_{M F}(x) \text { or } A^{\mu}(x) \\
v_{A^{*}}(x) \leftrightarrow v_{A}(x) \equiv A_{N M F}(x) \text { or } A^{v}(x)
\end{array}\right.
$$

We focus only on piecewise-linear MFs, namely trapezoids and triangles, because they are very widely used, and state space sculpting theory [1] has (so far) only been developed for them.

A NMF is said to be valid if and only if $0 \leq \mu_{A^{*}}(x)+v_{A^{*}}(x) \leq 1$, which can be satisfied in different ways, i.e., there are different ways to define a NMF for a given MF, e.g., [5] and [25]. One approach is to specify $\pi_{A^{*}}(x)$ and to then compute $v_{A^{*}}(x)$, whereas another approach is to choose $v_{A^{*}}(x)$ so that the above constraint is satisfied, and (if desired) to then examine the resulting $\pi_{A^{*}}(x)$. We take the latter approach in this paper because it leads to NMFs that are also piecewise linear and are easy to define. More specifically, we adopt Mahapatra and Roy's $A_{M F} / A_{N M F}$ pair (the $A_{M F} / A_{N M F}^{1}$ pair in Fig. 1b) [25]:

$$
A_{M F}(x)=\left\{\begin{array}{ll}
\frac{x-b}{c-b} & \text { for } b \leq x \leq c \\
1 & \text { for } c \leq x \leq d \\
\frac{e-x}{e-d} & \text { for } d \leq x \leq e \\
0 & \text { otherwise }
\end{array} \quad A_{N M F}^{1}(x)= \begin{cases}\frac{c-x}{c-a} & \text { for } a \leq x \leq c \\
0 & \text { for } c \leq x \leq d \\
\frac{x-d}{f-d} & \text { for } d \leq x \leq f \\
1 & \text { otherwise }\end{cases}\right.
$$

Note that the trapezoid MF in (4) reduces to a triangle MF when $c=d \in[b, e]$.

Theorem 1: (a) $A_{N M F}=A_{N M F}^{1}$ in (4) is a valid NMF, and (b)

The sloping legs of $A_{N M F}^{1}$ and $A_{M F}$ intersect below the grade of 0.5 .

Proofs of these results do not appear in [25] and are included in our Appendix A. The second part of Theorem 1 provides a simple way to quickly "see" if $A_{N M F}^{1}$ is valid. Observe that $A_{M F}$ in (4) is described by four parameters $\{b, c$,

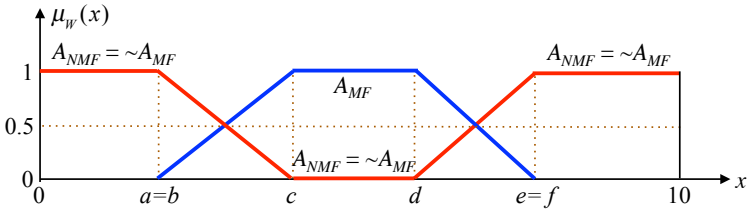

(a)

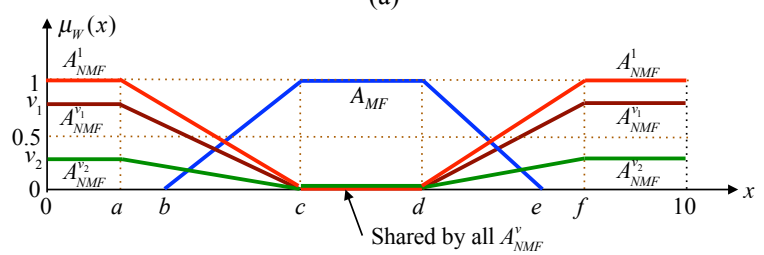

(b)

Fig. 1. (a) $A_{M F} / \sim A_{M F}$ and (b) $A_{M F} / A_{N M F}^{v}$ in which $A_{M F}$ (blue) is the same for all $A_{M F} / A_{N M F}^{v}$ pairs.

$d$, e\} and that $A_{N M F}^{1}$ is described by only two new parameters $\{a, f\}$.

$A_{N M F}^{1}$ in (4) is normal; however (also, not in [25]) it can be easily generalized to non-normal NMFs $A_{N M F}^{v}$, i.e.:

Corollary 1: Let $v$ be a scale factor, where $0<v \leq 1$; then $v A_{N M F}^{1} \equiv A_{N M F}^{v}$ (which is described by three new parameters, $a$, $f$ and $v$; see Fig. $1 \mathrm{~b}$ ) is a valid NMF, where $A_{N M F}^{1}$ is in (4).

Proof: Because $A_{N M F}^{1}$ is a valid NMF (Theorem 1) and $0<v \leq 1, \quad 0<A_{M F}+v A_{N M F}^{1} \leq A_{M F}+A_{N M F}^{1} \leq 1$. Consequently $v A_{N M F}^{1} \equiv A_{N M F}^{v}$ is also a valid NMF.

Corollary 2: When $a=b, e=f$ and $v=1$ then $A_{N M F}^{1}=\sim A_{M F}$, i.e., the NMF is the complement of the $M F$.

Proof: This is obvious from Figs. $1 \mathrm{~b}$ and $1 \mathrm{a}$.

Much of this paper focuses on this case because the parameters that define $A_{M F}$ also define $\sim A_{M F}$.

Definition 1: Points at which a MF or a NMF change its slope are called MF or NMF kinks. In this paper, to keep things relatively simple, it is assumed that such kinks only occur when a (membership) grade is $v$ or zero.

Observe, from Figs. 1a,b, that: (a) when $A_{N M F}=\sim A_{M F}$, the kinks of $\sim A_{M F}$ at zero (unity) grade are located at $x$-values where the kinks of $A_{M F}$ at unity (zero) grades are located; and, for all $0<v \leq 1$, (b) the two kinks of $A_{N M F}^{v}$ and $\sim A_{M F}$ at grade zero occur at the same points, $x=c, d$; and, (c) the two kinks of $A_{N M F}^{v}$ at grade $v$ occur at the same points, $x=a, f$, and these locations are different from the comparable two kink locations for $\sim A_{M F}$, which occur at $x=b, e$.

\section{B. Rule-Based Intuitionistic Fuzzy Systems}

This paper focuses for the most part only on type-1 I-fuzzy systems; hence, we omit "type-1" in our further descriptions. 


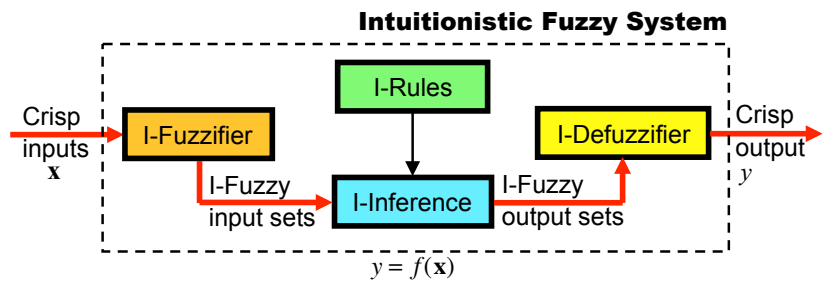

Fig. 2. I-fuzzy system [22].

A rule-based I-fuzzy system contains four components-Irules, I-fuzzifier, I-inference (engine), and I-defuzzifier - that are interconnected as shown in Fig. 2. Once the I-rules have been established, the I-fuzzy system can be viewed as a mapping from inputs to outputs, and this mapping can be expressed quantitatively as $y=f(\mathbf{x})$.

\section{I-Rules}

An I-fuzzy system uses two subsets of rules, $M$ MF rules and $M$ NMF rules [5], which collectively are its I-rules and are stated in (5) for an I-TSK fuzzy system [since sculpting the state space focuses only on the antecedents, the results in the rest of this paper are also valid for an I-Mamdani fuzzy system for which $y_{k}^{\mu}=b_{k}^{\mu}$ and $\left.y_{k}^{v}=b_{k}^{v}\right]$. In (5), $k=1, \ldots, M$, $x_{i} \in X_{i} \in \mathbb{R}(i=1, \ldots, p)$, and the MFs (NMFs) in $R_{k}^{\mu}\left(R_{k}^{v}\right)$ are all given by (4) in which $x$ is replaced by $x_{i}$. A TSK fuzzy system only uses the $R_{k}^{\mu}$ rules.

$$
\left\{\begin{aligned}
R_{k}^{\mu}: & \text { IF } x_{1} \text { is } A_{1 k}^{\mu} \text { and } \cdots \text { and } x_{p} \text { is } A_{p k}^{\mu}, \\
& \text { THEN } y_{k}^{\mu}=\sum_{i=1}^{p} w_{i k}^{\mu} x_{i}+b_{k}^{\mu} \\
R_{k}^{v}: & \text { IF } x_{1} \text { is } A_{1 k}^{v} \text { and } \cdots \text { and } x_{p} \text { is } A_{p k}^{v}, \\
& \text { THEN } y_{k}^{v}=\sum_{i=1}^{p} w_{i k}^{v} x_{i}+b_{k}^{v}
\end{aligned}\right.
$$

\section{I-Firing Levels in I-Fuzzy Systems}

For an I-fuzzy system, T1 fuzzy logic principles are used to map I-fuzzy input sets in $X_{1} \times \cdots \times X_{p}$ that flow through a set of I-rules into a crisp output, $y$. We assume singleton fuzzification, although the approach that is taken herein is conceptually the same regardless of the nature of the fuzzifier.

It is well known that, for $\mathrm{T} 1$ singleton fuzzification, when $\mathbf{x}=\mathbf{x}^{\prime}$ the firing levels for each subset of I-rules, are (e.g., [26], [27] $)(k=1, \ldots, M)$ :

$$
\left\{\begin{array}{l}
\text { MF firing levels: } \quad f_{k}^{\mu}\left(\mathbf{x}^{\prime}\right)=T_{i=1}^{p} A_{i k}^{\mu}\left(x_{i}^{\prime}\right) \\
\text { NMF firing levels: } f_{k}^{v}\left(\mathbf{x}^{\prime}\right)=T_{i=1}^{p} A_{i k}^{v}\left(x_{i}^{\prime}\right)
\end{array}\right.
$$

In (6), $T$ denotes a t-norm, usually the minimum or product. Observe that in (6), $\mathbf{x}^{\prime}$ is processed twice nonlinearly, whereas for a TSK fuzzy system ( $R_{k}^{\mu}$ rules) $\mathbf{x}^{\prime}$ is only processed once non-linearly.

Definition 2: An I-firing level $\left[f_{k}^{\mu}\left(\mathbf{x}^{\prime}\right)\right.$ or $\left.f_{k}^{v}\left(\mathbf{x}^{\prime}\right)\right]$ contributes to its output only if it is non-zero. This occurs in
$X_{1} \times \cdots \times X_{p}$ when the MFs of all $R_{k}^{\mu}$ - or $R_{k}^{v}$ - antecedents are simultaneously non-zero (i.e., active and firing).

\section{E. I-Defuzzification}

In an I-fuzzy system, after the I-firing levels have been computed there can be different ways to use them to obtain its final output by means of I-defuzzification (e.g., [5], [28]). One approach obtains $y\left(\mathbf{x}^{\prime}\right)$ by aggregating the $M \mathrm{MF}$ and $M$ NMF firing levels simultaneously, as one would do in a TSK fuzzy system. Another approach obtains $y^{\mu}\left(\mathbf{x}^{\prime}\right)$ for the MF subsystem and $y^{v}\left(\mathbf{x}^{\prime}\right)$ for the NMF subsystem, and then computes $y\left(\mathbf{x}^{\prime}\right)=(1-\beta) y^{\mu}\left(\mathbf{x}^{\prime}\right)+\beta y^{v}\left(\mathbf{x}^{\prime}\right)$. The results that are presented below apply to both approaches.

\section{F. Rule Partitions for TI and I-Fuzzy Systems}

One of the important things learned from [1, Section III] is that first- and second-order rule partitions of $X_{1} \times \cdots \times X_{p}$ are completely determined by the respective rule partitions of each $(i=1, \ldots, p) X_{i}$ separately, because, when minimum or product t-norms are used, if even one component of a rule's firing level is zero then that rule does not contribute to the output of the fuzzy system [e.g., $\min \left(\operatorname{any} A_{i k}^{\mu}=0\right.$, all other $\left.A_{i k}^{\mu}\right)=0 \quad$ and $\quad$ product $\left(\right.$ any $A_{i k}^{\mu}=0$, all other $\left.\left.A_{i k}^{\mu}\right)=0\right]$. This carries over as well from fuzzy systems to I-fuzzy systems [see (6)].

Definition 3: [1] In a T1 or I-fuzzy system, a first-order rule partition of $X_{i}$ is a collection of non-overlapping intervals in $X_{i}$, in each of which the same number of same rules is fired whose firing levels contribute to the output of that system.

Definition 4: [1] In a T1 or I-fuzzy system, a second-order rule partition line of $X_{i}$ occurs where the slope of the MF of a FS that is associated with $x_{i}$ changes its formula within a T1 first-order rule partition of $X_{i}$.

Rule partitions sculpt the state space into hyper-rectangles within each of which resides a different nonlinear function. First-order rule partitions provide a coarse sculpting whereas second-order rule partitions provide a fine sculpting.

Many examples of first- and second-order rule partitions for fuzzy systems are in [1] and its Supplementary Material (SM). For the convenience of the reader, Section I of the SM to the present paper contains six tables from [1] that provide notations used in first- and second-order rule partitions as well as procedures for establishing them.

\section{FIRST-ORDER RULE PARTITIONS FOR I-FUZZY SYSTEMS}

Because an I-fuzzy system is comprised of MF and NMF subsystems, we begin by explaining how first-order (MF and NMF) rule partitions are obtained for them, after which we explain how those two kinds of partitions are combined to obtain first-order rule partitions for the I-fuzzy system.

Assumption A: All results and examples herein are for $Q$ MFs: left and right shoulders and $Q-2$ interior trapezoidal 
MFs (not necessarily symmetrical), where each MF intersects only its neighboring left and right MFs once.

Examples in which a MF intersects more than one of its immediate neighboring left or right MFs are given in the SM, (Section II, Examples SM-3 and SM-4).

\section{A. First-Order MF Rule Partitions for One Variable}

First-order MF rule partition lines occur where a MF first goes from zero to a non-zero value (when a MF becomes "active"), and are located at grade-zero MF kinks.

Example 1: [1] Consider $x_{i} \in[0,10]$ covered by the three $\mathrm{T} 1$

FSs in Fig. 3a, for which there are three MF rules whose antecedents are $R_{1}^{M F}$ :IF $x_{i}$ is $L, R_{2}^{M F}:$ IF $x_{i}$ is $M$, and, $R_{3}^{M F}$ : IF $x_{i}$ is $H$. The orange circles in Fig. 3a are located at gradezero MF kinks. The first-order MF rule partition lines are drawn below the orange circles, and extend through two rows. In the first row, in " $m a$ " " $m$ " is the number of fired rules, and "a" is short for "active." In the second row, in " $\mathrm{Nm}$ " "l" is the numerical name for the first-order MF rule partition, and " $m$ " is the number of active rules in that first-order MF rule partition, e.g., $4 \backslash 2$ is partition 4 in which 2 rules are active. Observe that there are five first-order MF rule partitions, named $1,2, \ldots$ and $5 ; 1$ rule is fired in 1,2 rules are fired in 2, $\ldots$ and 1 rule is fired in 5 .

Other examples of first-order MF (and NMF) rule partitions for one variable are given in Section II of the SM.

The partition counts in Fig. 3a can be generalized to $Q$ MFs.

Theorem 2: Under Assumption A, if the $Q$ MFs collectively have $K_{0}$ kinks at membership grade zero then there are $K_{0}+1=2 Q-1$ first-order MF rule partitions.

The proof of this theorem appears after the statement of Theorem 3, because the proofs of both theorems are so similar.

Theorem 2 is very useful if one only wants to know the total number of first-order MF rule partitions.

\section{B. First-Order NMF Rule Partitions for One Variable}

To construct first-order NMF rule partition lines, it is expedient to first draw each NMF (e.g., $\sim L$, the complement of $L$ ) directly above one another, putting the NMF for the left(right-) most MF at the top (bottom) of the stack. They are not all put on the same plot because they overlap a lot at membership grade unity.

First-order NMF rule partition lines occur where a NMF first goes from zero to a non-zero value (which is when a NMF becomes "active"). These lines are located at grade-zero NMF kinks.

Example 2: This is a continuation of Example 1. The orange circles in Fig. $3 \mathrm{~b}$ are located at grade-zero NMF kinks. The first-order NMF rule partition lines are drawn below the orange circles, and extend through two rows. Observe that in Fig. $3 b$ there are five first-order NMF rule partitions, named 1, $2, \ldots$ and 5 , where 2 rules are fired in 1, 3 rules are fired in 2, $\ldots$ and 2 rules are fired in 5. Comparing Figs. 3a and 3b, observe that more rules are fired in each of the first-order $N M F$ rule partitions than are fired in the first-order MF rule partitions.

The partition counts in Fig. 3b can be generalized to $Q$ MFs.

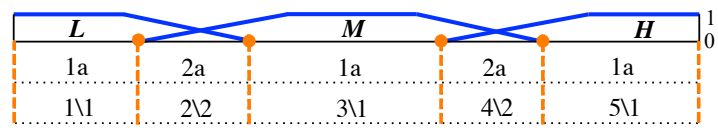

(a)

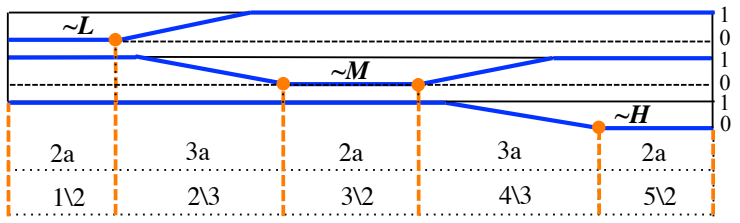

(b)

Fig. 3. First-order (a) Example $1 \mathrm{MF}$ and (b) Example $2 \mathrm{NMF}$ rule partitions for one variable, $Q=3$.

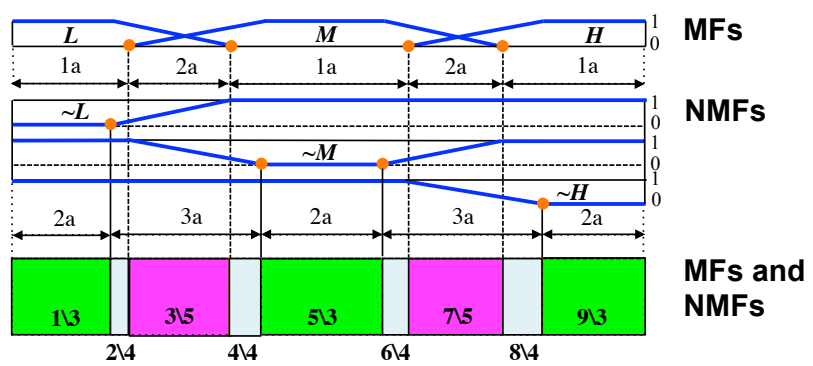

Fig. 4. Example 3 total number of first-order rule partitions in a onevariable I-fuzzy system, $Q=3$.

Theorem 3: Under Assumption A, if the $Q$ MFs collectively have $K_{1}$ kinks at membership grade unity then there are $K_{1}+1=2 Q-1$ first-order NMF rule partitions.

Theorem 3 is very useful if one only wants to know the total number of first-order NMF rule partitions.

Proof of Theorems 2 and 3: Shoulder MFs (or NMFs) have one kink at grade zero, whereas interior MFs (or NMFs) have two kinks at grade zero. Consequently, two shoulder and $Q-2$ interior MFs (or NMFs) have a total of $K_{0}\left(\right.$ or $\left.K_{1}\right)=$ $2 Q-2$ kinks at grade zero, which lead to $K_{0}+1$ (or $K_{1}+1$ ) $=2 Q-1$ first-order MF (or NMF) rule partitions (one partition occurs between the origin and the first kink).

Corollary 3: Under Assumption A, if $K_{0}=K_{1} \equiv K$, then the numbers of first-order MF and NMF rule partitions are the same, namely $K+1=2 Q-1$.

Proof: This follows from Theorems 2 and 3 when $K_{0}=K_{1} \equiv K$.

Examples 1 and 2 satisfy the conditions of Corollary 2, which is why their numbers of first-order MF and NMF rule partitions are the same.

\section{First-Order Rule Partitions for a One Variable I-Fuzzy System}

For each $x_{i}$, a certain number of MF and NMF rules are fired (active), so that the total number of fired rules in a onevariable I-fuzzy system is the sum of those two numbers, each of which can be read off from MF and NMF first-order rule partition figures.

Example 3: This is a continuation of Examples 1 and 2. Fig. 4 depicts MF, NMF and combined MF and NMF first-order 
rule partitions when $Q=3$. To determine the number or rules fired, stated in the bottom row of Fig. 4, project a vertical line upwards from a partition and add the two "a" numbers that it intersects. Observe that the one variable I-fuzzy system has nine first-order rule partitions (each with either three, four or five fired rules in it), which is almost double the number of first-order MF (or NMF) rule partitions in a MF (or NMF) fuzzy system (which is 5).

The extensions of Fig. 4 from $Q=3$ to other values of $Q$ are also given in Section II of the SM.

From all of these examples one concludes that there is a lot more coarse-sculpting going on even in a one-variable I-fuzzy system than in a TSK fuzzy system.

The partition counts in Fig. 4 can be generalized to $Q$ MFs.

Theorem 4: Under Assumption A, in a one-variable I-fuzzy system: (a) there are 4Q-3 first-order rule partitions, and (b) if the MFs collectively have $K_{0}$ kinks at grade zero and $K_{1}$ kinks at grade unity, then there will be $K_{0}+K_{1}+1$ first-order rule partitions.

Proof: (a) From the proof of Theorems 2 and 3, two shoulder and $Q-2$ interior MFs or NMFs collectively have a total of $4 Q-4$ kinks at grade zero. These lead to $4 Q-3$ firstorder rule partitions in a one-variable I-fuzzy system (one partition occurs between the origin and the first kink).

(b) This follows from the construction procedure for the first-order rule partitions in a one-variable I-fuzzy system, e.g. see Fig. 4. Observe that $K_{0}$ dashed lines are drawn downwards from the $K_{0}$ MF kinks at MF grade zero, and $K_{1}$ dashed lines are drawn downwards from the $K_{1}$ NMF kinks at NMF grade zero. Doing this leads to $K_{0}+K_{1}+1$ rectangles in the bottom portion of these figures, and those rectangles are the first-order rule partitions in a one-variable I-fuzzy system.

This theorem provides two ways to find the total number of first-order rule partitions in a one-variable I-fuzzy system.

Example 4: Using results from Example 3 and Theorem 4 $(Q=3), 4 Q-3=9$, which is correct. Fig. 3a reveals that $K_{0}=K_{1}=4$, hence $K_{0}+K_{1}+1=9$, which is also correct. More examples that demonstrate the correctness of $4 Q-3$ $=K_{0}+K_{1}+1$ are in Section III of the SM.

A critic of an I-fuzzy system may argue that, of course such a system has more first-order rule partitions than does a MF fuzzy system, because it has twice as many rules. The following theorem demonstrates that it is possible to level the playing field for these two kinds of fuzzy systems, so that they both have exactly the same number of such partitions.

Theorem 5: Under Assumption A, a one-variable TSK fuzzy system that uses $Q_{M F}$ MFs, and a one-variable I-fuzzy system that uses $Q_{I}$ MFs, have exactly the same number of first-order rule partitions (their sizes and locations may be different), if

$$
Q_{M F}=2 Q_{I}-1
$$

Proof: From Theorems 2 and 4, the numbers of respective first-order rule partitions are $2 Q_{M F}-1$ and $4 Q_{I}-3$. (7) is obtained by equating these two numbers and solving for $Q_{M F}$.

Although it is possible to level the playing field as just described, this comes at a cost to the TSK fuzzy system, in that it will have many more design parameters to tune than does the I-fuzzy system.

Example 5: When $Q_{I}=3$, a six-rule I-fuzzy system, whose MFs are the ones depicted in Fig. 3a, will have (assuming $M$ is not symmetrical) eight MF parameters and, if its TSK rule consequents are constants, six consequent parameters, for a total of 14 design parameters, whereas (using (7)) a five-rule TSK fuzzy system, whose five MFs include two shoulders and three interior trapezoids, will have $16 \mathrm{MF}$ parameters, and, if its TSK rule consequents also are constants, five consequent parameters, for a total of 21 design parameters - $50 \%$ more parameters to tune; for $Q_{I}=4$ an eight-rule I-fuzzy system will have a total of 20 design parameters, whereas a seven-rule MF fuzzy system will have a total of 31 design parameters, slightly more than $50 \%$ as many design parameters to tune.

\section{First-Order MF Rule Partitions for Two Variables}

The construction procedure for creating first-order MF rule partitions for two variables is unchanged from the one that is given in Table III of [1], and is based on constructing the firstorder MF rule partitions for $X_{1}$ and $X_{2}$, and then combining those results. See Section I of the SM (Table SM-III).

Example 6: This is a continuation of Example 1 (Fig. 3a). Its results are taken from [1] and are repeated here because they are used below in Section F. The first-order MF rule partition diagram for $X_{1} \times X_{2}$ is depicted in Fig. 5. The number in each rectangle denotes its number of active MF rules, and is obtained by multiplying the comparable numbers for $X_{1}$ and $X_{2}$ that are given along the horizontal and vertical axes, respectively. The total number of first-order MF rule partitions is the product of the total number of such partitions (five) for each of the two variables, and is 25.

Theorem 6: Under Assumption A, if $X_{1}$ and $X_{2}$ are covered by $Q_{1}$ and $Q_{2}$ MFs, respectively, with $K_{0}\left(X_{1}\right)$ and $K_{0}\left(X_{2}\right)$ kinks at grade zero, then there will be $\left[K_{0}\left(X_{1}\right)+1\right]$ $\left[K_{0}\left(X_{2}\right)+1\right]$ first-order MF rule partitions of $X_{1} \times X_{2}$.

The proof of this theorem appears after the statement of Theorem 7, because the proofs of both theorems are so similar.

Theorem 6 does not appear in [1] and is very useful when one only wants to know the total number of first-order MF rule partitions of $X_{1} \times X_{2}$

\section{E. First-Order NMF Rule Partitions for Two Variables}

The construction procedure for creating first-order NMF rule partitions for two variables also uses the one that is given in Table III of [1], and is based on constructing the first-order NMF rule partitions for $X_{1}$ and $X_{2}$, and then combining those results. 


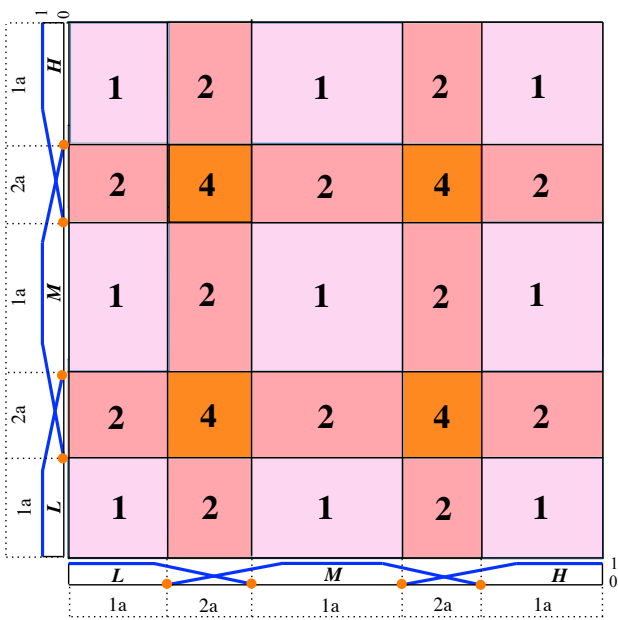

Fig. 5. Example 6 first-order MF rule partitions for two variables, $Q$ $=3$.

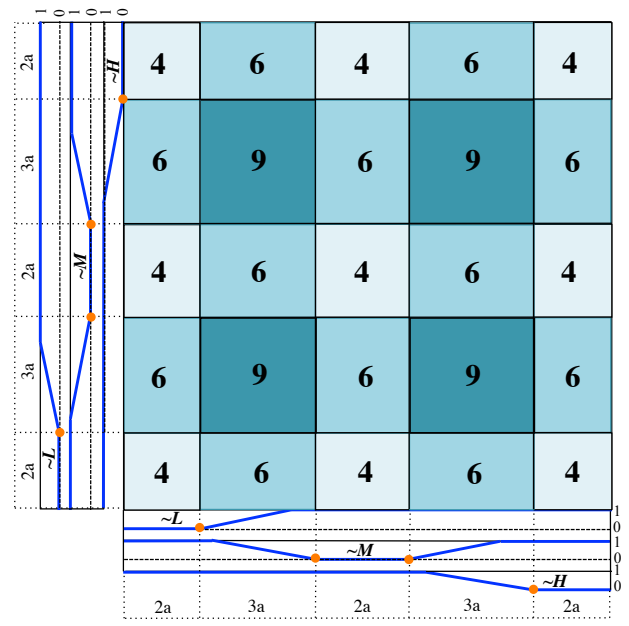

Fig. 6. Example 7 first-order NMF rule partitions for two variables, $Q$ $=3$.

Example 7: This is a continuation of Example 2 (Fig. 3b). The first-order NMF rule partition diagram for $X_{1} \times X_{2}$ is depicted in Fig. 6. The number in each rectangle denotes its number of active NMF rules, and is obtained by multiplying the comparable numbers for $X_{1}$ and $X_{2}$ that are given along the horizontal and vertical axes, respectively. The total number of first-order NMF rule partitions is the product of the total number of such partitions (five) for each of the two variables, and is 25 .

Comparing Figs. 5 and 6, observe that, just as for one variable, more rules are fired in each of the first-order NMF rule partitions than are fired in the first-order MF rule partitions.

Theorem 7: Under Assumption A, if $X_{1}$ and $X_{2}$ are covered by $Q_{1}$ and $Q_{2}$ MFs, respectively, with $K_{1}\left(X_{1}\right)$ and $K_{1}\left(X_{2}\right)$ kinks at grade unity, then there will be $\left[K_{1}\left(X_{1}\right)+1\right]$ $\left[K_{1}\left(X_{2}\right)+1\right]$ first-order NMF rule partitions of $X_{1} \times X_{2}$.

Theorem 7 is very useful when one only wants to know the total number of first-order NMF rule partitions on $X_{1} \times X_{2}$.
Proof of Theorems 6 and 7: Theorem 6 (7) follows from Theorem 2 (3) in which $K_{0}\left(K_{1}\right)$ is replaced by $K_{0}\left(X_{1}\right)$ $\left[K_{1}\left(X_{1}\right)\right]$ for $X_{1}$ and $K_{0}\left(X_{2}\right)\left[K_{1}\left(X_{2}\right)\right]$ for $X_{2}$, after which Eq. (6) in [1] is used. That equation is: $N_{*}^{1}\left(X_{1}, \ldots, X_{p}\right)=N_{*}^{1}\left(X_{1}\right) \cdots N_{*}^{1}\left(X_{p}\right)$, where $N_{*}^{1}\left(X_{j}\right)$ is the number of first-order rule partitions of $X_{j}$ and $*$ is T1 or IT2.

Corollary 4: Under Assumption A, if $K_{0}=K_{1} \equiv K$, then the numbers of first-order MF and NMF rule partitions on $X_{1} \times X_{2}$ are the same, namely $(K+1)^{2}=(2 Q-1)^{2}$.

Proof: This follows from Theorems 6 and 7 when $K_{0}=K_{1} \equiv K$.

Examples 6 and 7 satisfy the conditions of Corollary 3 $(K=4)$, which is why their numbers of first-order MF and NMF rule partitions are the same.

F. First-Order MF and NMF Rule Partitions for More Than Two Variables

Theorem 8: Under Assumption A and $K_{0}=K_{1} \equiv K$, let $N_{M F}^{1}\left(X_{1}, \ldots, X_{p}\right)$ and $N_{N M F}^{1}\left(X_{1}, \ldots, X_{p}\right)$ respectively denote the total number of first-order MF and NMF rule partitions of $X_{1} \times \cdots \times X_{p}$. Then

$$
N_{M F}^{1}\left(X_{1}, \ldots, X_{p}\right)=N_{N M F}^{1}\left(X_{1}, \ldots, X_{p}\right)=(2 Q-1)^{p}=(K+1)^{p}
$$

Proof: This is the extension of Corollary 4 from two to $p$ variables.

The extension of (8) to the case when $Q$ is not the same for each $X_{i}$ is straightforward (use Theorems 6 and 7).

\section{G. First-Order Rule Partitions in a Two-Variable I-Fuzzy System}

For each $\left(x_{1}, x_{2}\right)$ pair, a certain number of MF and NMF rules are fired (active), so that the total number of fired rules in a two-variable I-fuzzy system is the sum of those two numbers, each of which, e.g. for $Q=3$, is read off from figures like Figs. 5 or 6.

Example 8: This is a continuation of Examples 6 and 7. Fig. 7 depicts MF, NMF and combined MF and NMF first-order rule partitions when $Q=3$. Exactly how this figure was obtained from Figs. 5 and 6 is explained in Section III of the SM. Observe that this two variable I-fuzzy system has 81 firstorder rule partitions, whereas the "traditional" two-variable TSK fuzzy system only has 25 first-order rule partitions (Fig. 5 ), a more than tripling of the number of first-order rule partitions.

Obtaining a figure like the one in Fig. 7 is extremely tedious. The following is a way to bypass this construction if one only wants to know the total number of first-order rule partitions in a two-variable I-fuzzy system.

Theorem 9: Under Assumption A for $X_{1}$ and $X_{2}$, there are $(4 Q-3)^{2}$ first-order rule partitions in a two-variable I-fuzzy system. 


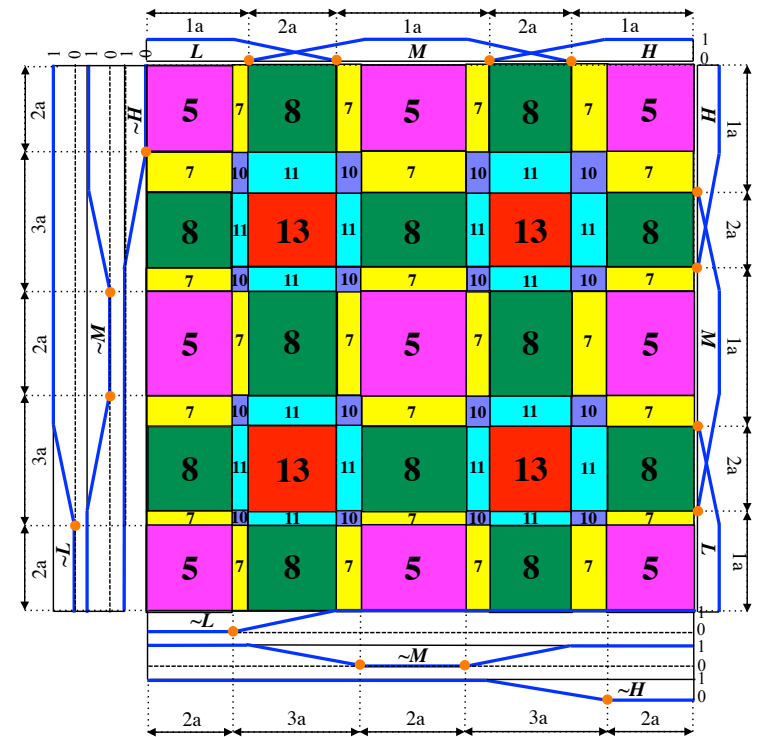

Fig. 7. Example 8 total number of first-order rule partitions in a twovariable I-fuzzy system, $Q=3$.

Proof: This follows from Theorem 4 applied to two variables.

Example 9: When $Q=3,(4 Q-3)^{2}=9^{2}=81$, which agrees with the results in Example 8.

H. Total Number of First-Order Rule Partitions in a pVariable I-Fuzzy System

Theorem 10: Under Assumption A for each of $p$ variables, when $K_{0}=K_{1}=K$ the number of first-order rule partitions in a $p$-variable I-fuzzy system, $N_{I}^{1}\left(X_{1}, \ldots, X_{p}\right)$, is:

$$
N_{I}^{1}\left(X_{1}, . ., X_{p}\right)=(4 Q-3)^{p}
$$

Proof: This follows from Theorem 4 applied to $p$ variables.

For a TSK fuzzy system, there are $\left[\right.$ see (8)] $(2 Q-1)^{p}$ first order MF rule partitions of $X_{1} \times \cdots \times X_{p}$. Comparing $(2 Q-1)^{p}$ and $(4 Q-3)^{p}$, observe that, as $Q$ increases:

$$
\left(\frac{4 Q-3}{2 Q-1}\right)^{p}=\left(2-\frac{1}{2 Q-1}\right)^{p} \rightarrow 2^{p}
$$

This means that, as $Q$ increases, there will be a factor of approximately $2^{p}$ more first-order rule partitions of $X_{1} \times \cdots \times X_{p}$ in an I-fuzzy system than the number of such partitions in a TSK fuzzy system, which leads to our conjecture that the hugely-greater coarse sculpting of the state space by an I-fuzzy system provides it with the potential to outperform a TSK fuzzy system.

\section{SECOND-ORder Rule PARTITIONS FOR I-FuZZY Systems}

Second-order rule partitions for an I-fuzzy system can be found from $\mathrm{MF}$ and NMF second-order rule partition diagrams. This section is still for $A_{N M F}^{1}=\sim A_{M F}$.

\section{A. Second-Order MF Rule Partitions for One Variable}

Second-order MF rule partition lines occur where the slope of a MF changes within a first-order MF rule partition. These lines occur at grades zero and unity MF kinks, but because MF kinks at grade zero have already contributed the first-order partition lines, new lines are only needed at the unity-grade kinks.

Example 10: This is a continuation of Example 1. Fig. 8a begins with Fig. $3 \mathrm{a}$ after which dotted vertical lines are drawn at grade-unity MF kinks, indicated with brown filled-in circles. The second-order MF rule partitions are drawn below the brown circles and extend into one row. In that row, regarding the notation $\lambda m: n, l$ is the numerical name for the first-order MF rule partition; $m$ is the number of rules fired in that partition; and, $n$ are the number of second-order MF rule partitions in it, e.g., $3 \backslash 1: 3$ denotes first-order MF rule partition " 3 ", in which " 1 " rule is fired and in which there are " 3 " second-order MF rule partitions. Observe that there are 2 second-order MF rule partitions in 1, 0 second-order MF rule partitions in $2, \ldots$ and 2 second-order MF rule partitions in 5. The total number of second-order MF rule partitions is seven.

\section{B. Second-Order NMF Rule Partitions for One Variable}

Second-order NMF rule partition lines occur where the slope of a NMF changes within a first-order NMF rule partition. These lines occur at grades zero and unity NMF kinks, but because grade-zero NMF kinks have already contributed the first-order partition lines, new lines are only needed at the grade-unity kinks.

Example 11: This is a continuation of Example 2. Fig. 8b begins with Fig. $3 \mathrm{~b}$ after which dotted vertical lines are drawn at grade-unity kinks, also indicated with brown filled-in circles. The second-order NMF rule partitions are drawn below the brown circles and extend into one row. The total number of second-order NMF rule partitions is six. In this example the number of second-order NMF rule partitions is less than the number of second-order MF rule partitions. Examples SM-6 and SM-7 in the SM demonstrate that it is also possible for the number of second-order NMF rule partitions to be greater than or equal to the number of secondorder MF rule partitions. Such results depend on the relative locations of the grades zero and unity MF and NMF kinks.

\section{Second-Order Rule Partitions for a One-Variable I-Fuzzy System}

Comparing Figs. 8a and b observe that first-order MF lines become second-order NMF lines, and second-order MF lines become first-order NMF lines.

Examining the last row of Fig. 4, and making use of the just-stated fact, it should be clear that it is not possible to show the second-order rule partition lines on this figure, because they will lie on top of the first-order MF and NMF lines. This does not mean that second-order rule partitions vanish in the Ifuzzy system. They still exist, but when $A_{N M F}=\sim A_{M F}$ they can only be observed on the individual second-order MF and NMF rule partition diagrams, namely on figures like Figs. 8a and 8b. 


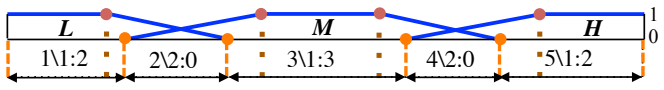

(a)

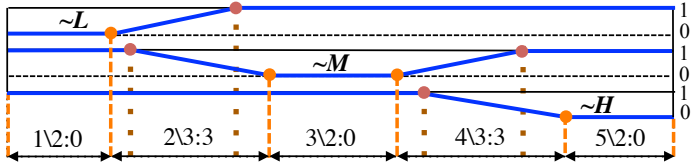

(b)

Fig. 8. Second-order (a) Example $10 \mathrm{MFs}$ and (b) Example $11 \mathrm{NMFs}$ rule partitions for one variable, $Q=3$.

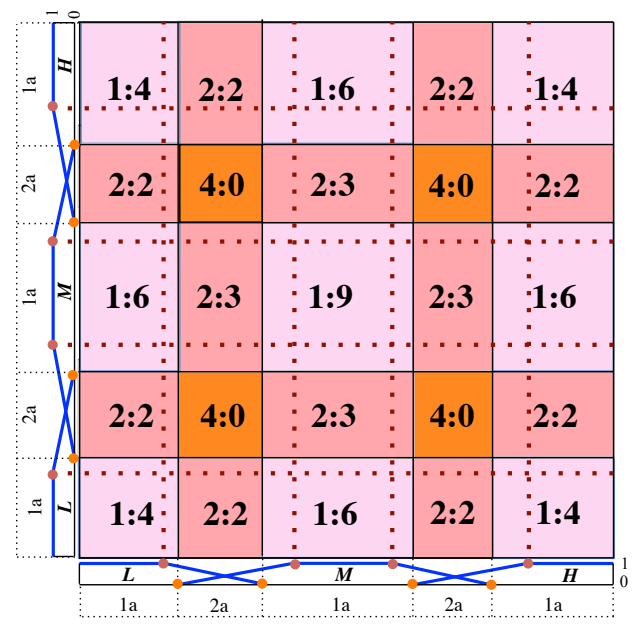

Fig. 9. Example 12 second-order MF rule partitions for two variables, $Q=3$.

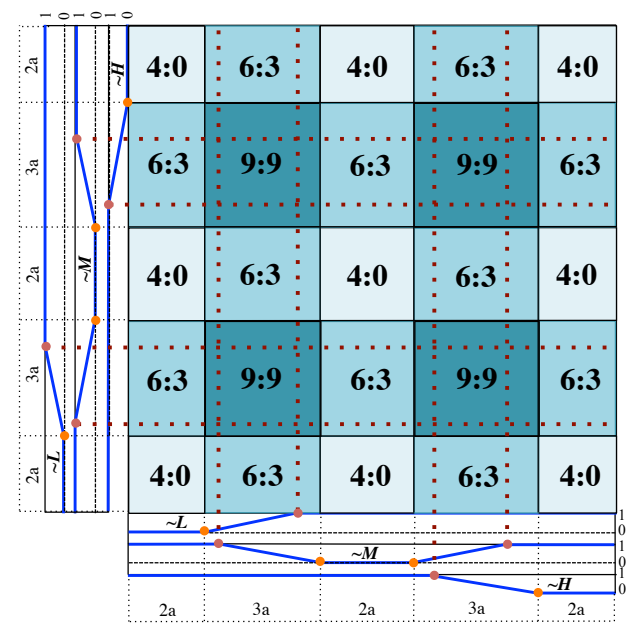

Fig. 10. Example 13 second-order NMF rule partitions for two variables, $Q=3$.

\section{Second-Order MF Rule Partitions for Two Variables}

The construction procedure for creating second-order MF rule partitions for two variables is unchanged from the one that is given in Table VI of [1], and begins with a first-order MF rule partition diagram. It is given in Table SM-VI in the $\mathrm{SM}$

Example 12: This is a continuation of Examples 6 and 10 when $Q=3$. Its results are taken from [1], and are repeated here because they are needed in Section F below. The secondorder MF rule partition diagram for $X_{1} \times X_{2}$ is depicted in Fig. 9. Note that regarding the notation $m: t$, that appears in each of the Fig. 9 rectangles, $m$ is the number of rules fired in that first-order MF rule partition; and, $t$ is the number of second-order MF rule partitions in it, e.g., 2:3 denotes " 2 " rules are fired and there are " 3 " second-order MF rule partitions. The total number of second-order MF rule partitions is obtained by counting and is 77 .

As is explained in [1, Section III.B], there is a formula for computing the total number of second-order MF rule partitions for $p$ variables, one that only needs information about the second-order MF rule partitions for each variable, and so it is practical. It is given in Section V of the SM.

\section{E. Second-Order NMF Rule Partitions for Two Variables}

The construction procedure for creating second-order NMF rule partitions for two variables is also the one that is given in Table VI of [1], and begins with a first-order NMF rule partition diagram.

Example 13: This is a continuation of Examples 7 and 11 when $Q=3$. The second-order NMF rule partition diagram for $X_{1} \times X_{2}$ is depicted in Fig. 10. The total number of second-order NMF rule partitions is again obtained by counting, and is 72 .

A formula for computing the total number of second-order NMF rule partitions for $p$ variables is also given in Section V of the SM.

\section{F. Second-Order Rule Partitions for a Two-Variable I-Fuzzy System}

Comparing Figs. 9 and 10 observe that once again firstorder MF lines become second-order NMF lines, and secondorder MF lines become first-order NMF lines. Consequently, it is not possible to show the second-order rule partition lines on Fig. 7, because they will lie on top of the first-order MF and NMF lines. This does not mean that second-order rule partitions have disappeared in the I-fuzzy system. They still exist, but, when $(i=1,2) \quad A_{N M F}\left(x_{i}\right)=\sim A_{M F}\left(x_{i}\right)$ they can only be observed on the individual second-order MF and NMF rule partition diagrams, namely on figures like Figs. 9 and 10.

\section{PARTITIONS FOR MORE GENERAL I-FuZZY SySTEMS}

This section extends the Sections III and IV results to $A_{M F} / A_{N M F}^{v}$ in (4), where $A_{N M F}^{v}=v A_{N M F}$ and $0<v \leq 1$ (see Fig. $1 \mathrm{~b}$ and Corollary 1$)$.

\section{A. First-Order Rule Partitions}

Theorem 11: All results that are given in Section III about first-order NMF or combined MF and NMF rule partitions are unchanged for all $A_{N M F}^{v}, 0<v \leq 1$.

Proof: First-order MF and NMF rule partitions are found by examining where kinks occur at grade zero, and this theorem is true because they occur at exactly the same points for all $A_{N M F}^{v}$ (see Fig. 1b).

Example 14: Fig. 11 gives first-order partition information for MFs, NMFs and both MFs and NMFs in a one-variable Ifuzzy system, when MFs and NMFs are given in (4), and $v=1$. Comparing Figs. 11 and 4, observe that: (1) The 


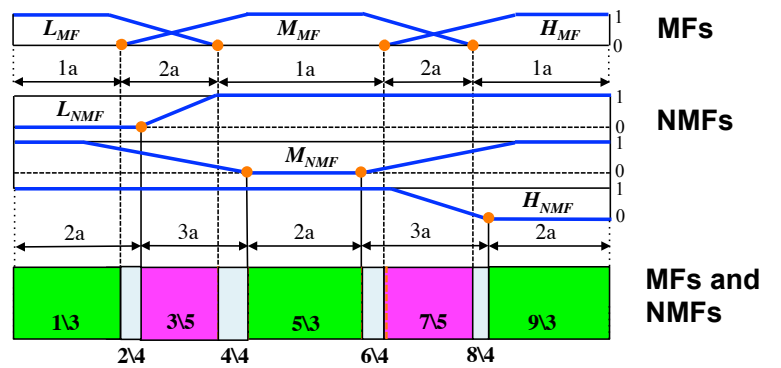

Fig. 11. Example 14 total number of first-order rule partitions in a one-variable I-fuzzy system, when $A_{N M F}^{v}=A_{N M F}^{1}$ ) and $Q=3$.

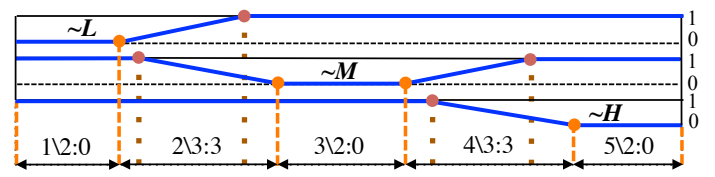

(a)

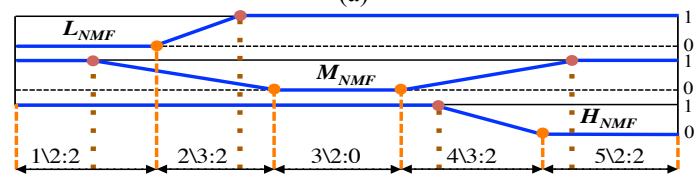

(b)

Fig. 12. Example 15 second-order NMF rule partitions for one variable, $Q=3$ : (a) $A_{N M F}=\sim A_{M F}$ and (b) $A_{N M F}^{v}=A_{N M F}^{1}$.

number of first-order NMF and combined MF and NMF rule partitions are unchanged; and, (2) It is only the sizes of some of the NMF and combined MF and NMF rule partitions that have changed.

\section{B. Second-Order Rule Partitions}

Theorem 12: Second-order rule partitions for $A_{N M F}^{v}$ $(0<v \leq 1)$ are different from those of $\sim A_{M F}$.

Proof: Second-order MF and NMF rule partitions are found by examining where kinks occur at grade unity, and they occur at exactly the same points for all $A_{N M F}^{v}$ (see Fig. 1b), but these points are different from those for $\sim A_{M F}$ (compare Figs. 1a and $1 \mathrm{~b}$ ), hence the truth of this theorem.

Example 15: Fig. 12a is a repeat of Fig. 8b, and Fig. 12b shows the second-order partition lines for the NMF portion of Fig. 11. Comparing Figs. 12a and 12b, observe that: (1) there are 8 (6) second-order NMF rule partitions in Fig. 12b (a); and, (2) The locations of some of the second-order NMF rule partitions in Fig. 12b are different from the locations in Fig. 12a. At last, we see a difference between using $\sim A_{M F}$ and $A_{N M F}^{1}$, namely, an I-fuzzy system that uses $A_{N M F}^{1}$ can achieve a finer sculpting of the state space than an I-fuzzy system that uses $\sim A_{M F}$.

Because second-order rule partitions are found by examining where grade-unity kinks occur, and they occur at exactly the same points for all $A_{N M F}^{v}$, the results shown in Fig. 12 for the locations and sizes as well as the number of fired rules in each partition are the same for all $A_{N M F}^{v}$. It seems,
TABLE I

EXAMPLE 16 FirST-AND SECOND-ORDER RULE PARTITIONS $(Q=3)$

\begin{tabular}{llll}
\hline \hline $\begin{array}{c}\text { Fuzzy } \\
\text { system }\end{array}$ & $\begin{array}{c}\text { Number of } \\
\text { variables }\end{array}$ & $\begin{array}{c}\text { Number of first- } \\
\text { order rule partitions }\end{array}$ & $\begin{array}{l}\text { Number of second- } \\
\text { order rule partitions }\end{array}$ \\
\hline T1 & 1 & 5 (Example 1) & 7 (Example 10) \\
IT2 & 1 & 5 [1, Example 1] & 9 [1, Example 3] \\
I- & 1 & 9 (Example 4) & 13 (Figs. 8a, b) \\
T1 & 2 & 25 (Example 6) & 77 [1, Example 4] \\
IT2 & 2 & $25[1$, Example 6] & 135 [1, Example 4] \\
I- & 2 & 81 (Example 8) & 149 (Figs. 9 and 10) \\
\hline \hline
\end{tabular}

therefore, that the only advantage for using $A_{N M F}^{v}, v \neq 1$, is one additional design degree of freedom, $v$.

\section{OBSERVATIONS}

Because the numbers of first- and second-order rule partitions only depend on the number of kinks at either zero or unity, or $Q$ (Theorems 2-4, 6-12), partition counts do not depend on (Assumption A) "... where each MF intersects only its neighboring left and right MFs once." They do depend on (Assumption A) "... for $Q$ MFs: left and right shoulders and Q-2 interior trapezoidal MFs (not necessarily symmetrical)". See, e.g. Examples SM-3 and SM-4 in the SM for further verification of this.

Although this paper has focused exclusively on T1 fuzzy systems, it is very interesting to compare the number of firstand second-order rule partitions for a T1, IT2 and I-fuzzy system.

Example 16: We do this for $Q=3$ in Table I. Observe that for one variable an IT2 (I-) fuzzy system has 5 (9) first-order and $9\left(13^{4}\right)$ second-order rule partitions, and for a two variables an IT2 (I-) fuzzy system has 25 (81) first-order and $135\left(149^{5}\right)$ second-order rule partitions. This (partition theory) suggests that an I-fuzzy system has the potential to outperform an IT2 fuzzy system, something that has already been demonstrated in [29] and [30], but for Gaussian T1 and IT2 MFs.

We conjecture (from partition theory) the following ordering for performance improvement for fuzzy systems:

T1 $<$ IT2 $<$ T1 Intuitionistic $<$ GT2 $<$ IT2 Intuitionistic

$<$ GT2 Intuitionistic

\section{CONCLUSIONS AND FUTURE WORK}

This paper has provided new application independent perspectives about the performance potential of an intuitionistic (I-) fuzzy system over a (classical TSK) fuzzy system, by extending sculpting the state space works from a fuzzy system to an I-fuzzy system. It has demonstrated that, for piecewise-linear MFs (trapezoids and triangles), an I-fuzzy system always has significantly more first-order rule partitions of the state space-the coarse sculpting of the state spacethan does a TSK fuzzy system, and that some I-fuzzy systems also have more second-order rule partitions of the state space - the fine sculpting of the state space - than does a TSK

\footnotetext{
${ }^{4}$ This number was obtained by adding the numbers of second-order rule partitions in Figs. $8 \mathrm{a}$ and $8 \mathrm{~b}$.

${ }^{5}$ This number was obtained by adding the numbers of second-order rule partitions in Figs. 9 and 10.
} 
fuzzy system. It is the author's conjecture that: It is the always significantly greater coarse (and possibly finer) sculpting of the state space that provides an I-fuzzy system with the potential to outperform a TSK fuzzy system.

Rule partition theory demonstrates the potential for improved performance, but it is the architecture of an I-fuzzy system that attempts to achieve this. Choosing NMFs as the complements of the MFs introduces no new design parameters; choosing them as in (4) introduces two new design parameters for each NMF; and choosing them by using a scaled version of (4) introduces yet one more design parameter.

Some open research questions and extensions to this paper are:

1) Study how much performance improvement occurs when one uses a NMF other than the complement of the MF. This may be application dependent.

2) Extend all results to IT2 I-fuzzy systems. We don't expect any surprises, i.e., an IT2 fuzzy system usually has the same number of first-order rule partitions as does a T1 fuzzy system (although they are of different sizes), but an IT2 fuzzy system usually has many more secondorder rule partitions than does a T1 fuzzy system. Consequently, an IT2 fuzzy system usually has a much greater fine sculpting capability of the state space than does a T1 fuzzy system. We expect comparable results between T1 and IT2 I-fuzzy systems.

3) Extend all results to GT2 I-fuzzy systems. Such systems have not appeared yet.

4) Extend all results to non-singleton T1 and IT2 I-fuzzy systems. Such systems also have not appeared yet.

5) Demonstrate (or not) the performance ordering for fuzzy systems that is given in (11).

6) Extend all sculpting results to Gaussian- (and bell-) shaped MFs. This needs to be done not only for I-fuzzy systems but also for all other kinds of fuzzy systems.

7) Study (suggested by a reviewer) the connections, and provide some new ones, between sculpting the state space and universal approximation for I-fuzzy systems.

8) Develop rule-partition mathematics. Drawing partition diagrams can be tedious. Mathematics for determining the number of first- and second-order rule partitions for any value of $Q$ and $p=1,2$ is already under development and will be reported on in another article.

9) Extend patch learning [31] (PL) to I-fuzzy systems. A patch is a first-order rule partition. PL consists of three steps: 1) train an initial global model using all training data; 2) identify from the initial global model the patches which contribute the most to the learning error, and train a (local) patch model for each such patch; and, 3) update the global model using training data that do not fall into any patch. [31] has demonstrated the effectiveness of PL on five regression problems, but to-date only for $\mathrm{T} 1$ fuzzy systems.

\section{APPENDIX A: PROOF OF THEOREM 1}

(a) $A_{N M F}^{1}$ is a valid NMF if

$$
A_{N M F}^{1}(x)+A_{M F}(x) \leq 1 .
$$

Examining Fig. 1b, it is clear that (A-1) is satisfied when $x \in[0, b], x \in[c, d]$ and $x \in[e, 10]$, so one only needs to see if (A-1) is satisfied for $x \in[b, c]$ and $x \in[d, e]$. The proof is only provided here for $x \in[b, c]$ and $v=1$, for which one must examine:

$$
(x-b) /(c-b)+(c-x) /(c-a) \stackrel{?}{\leq} 1
$$

It is straightforward to show, by means of simple algebra that (A-2) reduces to $x \leq c$ which is always true for $x \in[b, c]$; hence, (A-1) is satisfied when $x \in[b, c]$.

(b) Focusing on $x \in[b, c]$, one needs to first locate the intersection point of $A_{N M F}^{1}$ and $A_{M F}$, say $x^{*}$, which is found by solving the equation $\left(x^{*}-b\right) /(c-b)=\left(c-x^{*}\right) /(c-a)$, after which the intersection point, $A_{M F}\left(x^{*}\right)$, is computed, i.e. $x^{*}=\left(c^{2}-a b\right) /(2 c-a-b) \& A_{M F}\left(x^{*}\right)=(c-b) /(2 c-a-b)$.

To prove that $A_{M F}\left(x^{*}\right)<0.5$, assume $A_{M F}\left(x^{*}\right) \geq 0.5$ and show that this leads to a contradiction, i.e.,

$$
(c-b) /(2 c-a-b) \geq 0.5 \Rightarrow b-a \leq 0
$$

Examining Fig. $1 \mathrm{~b}$, observe that $b-a>0$; hence, (A-3) is false, which means that $A_{M F}\left(x^{*}\right)<0.5$ is true.

\section{ACKNOWLEDGMENT}

The authors would like to thank the reviewers of this paper who made very useful suggestions that have (hopefully) led to an improved version of this paper.

\section{REFERENCES}

[1] J. M. Mendel, "Explaining the performance potential of rule-based fuzzy systems as a greater sculpting of the state space," IEEE Trans. on Fuzzy Systems, vol. 26, pp. 2362-2373, August 2018.

[2] J. M. Mendel, "Comparing the performance potentials of interval and general type-2 rule-based fuzzy systems in terms of sculpting the state space," IEEE Trans. on Fuzzy Systems, vol. 27, pp. 58-71, Jan. 2019.

[3] J. M. Mendel, R. Chimatapu and H. Hagras, "Comparing the performance potentials of singleton and non-singleton type-1 and interval type-2 fuzzy systems in terms of sculpting the state space," IEEE Trans. on Fuzzy Systems, Early Access, June 2019.

[4] K. T. Atanassov, "Intuitionistic fuzzy sets," Fuzzy Sets and Systems, vol. 20, no. 1 , pp. 87-96, 1986.

[5] P. Hájek and V. Olej, "Defuzzification methods in intuitionistic fuzzy inference systems of Takagi-Sugeno type: The case of corporate bankruptcy prediction," In the Proc.of 11th Int. Conf. on Fuzzy Systems and Knowledge Discovery (FSKD 2014), Xiamen, China, pp. 240-244, 2014.

[6] P. Hájek and V. Olej, "Intuitionistic fuzzy neural network: The case of credit scoring using text information," in Engineering Applications of Neural Networks (L. Iliadis and C. Jayne, Eds.), Proc. of EANN, Sept. 2015, Rhodes, Greece, Springer, Cham Switzerland, pp. 337-346, 2015.

[7] D.-F. Li, "Multiattribute decision making models and methods using intuitionistic fuzzy sets," J. of Computer and System Sciences, vol. 70, no. 1, pp. 73-85, 2005.

[8] Z. Xu, "Some similarity measures of intuitionistic fuzzy sets and their applications to multiple attribute decision making," Fuzzy Optimization and Decision Making, vol. 6, no. 2, pp. 109-121, 2007.

[9] H.-W. Liu and G.-J. Wang, "Multi-criteria decision-making methods based on intuitionistic fuzzy sets," European J. of Operational Research, vol. 179, no. 1, pp. 220-233, 2007.

[10] J. Li and Z. Tian, "Intuitionistic fuzzy comprehensive evaluation in decision-making problem," in Proc. of IEEE 2010 Seventh Int'l. Conf. on Fuzzy Systems and Knowledge Discovery, vol. 1, pp. 203-206, 2010. 
[11] L. Zhang, "Multiple attributes group decision making under intuitionistic fuzzy preference settings," in Proc. of IEEE 2018 Chinese Control and Decision Conf., pp. 2202-2206, 2018.

[12] C.-L. Fan, Y. Song, Q. Fu, L. Lei and X. Wang, "New operators for aggregating intuitionistic fuzzy information with their application in decision making," IEEE Access, vol. 6, pp. 27,214-27,238, 2018.

[13] S. Maheshwari and A. Srivastava, "A new divergence measure for intuitionistic fuzzy sets and its application in medical diagnosis," in Proc. of IEEE 2015 Int'l. Conf. on Signal Processing and Communication, pp. 151-155, 2015.

[14] M. Wu, C. Zhou and K. Lin, "An intelligent TCM diagnostic system based on intuitionistic fuzzy set," in Proc. of IEEE Fourth Int'l. Conf. on Fuzzy Systems and Knowledge Discovery, vol. 1, pp. 91-95, 2007.

[15] S. K. De, R. Biswas and A. R. Roy, "An application of intuitionistic fuzzy sets in medical diagnosis," Fuzzy Sets and Systems, vol. 117, no. 2, pp. 209-213, 2001.

[16] A. Belsare, M. Mushrif and M. Pangarkar, "Breast epithelial duct region segmentation using intuitionistic fuzzy based multi-texture image map," in Proc. of IEEE 2017 14th India Council Int'l. Conf., pp. 1-6, 2017.

[17] P. Melo-Pinto, P. Couto, H. Bustince, E. Barrenechea, M. Pagola and J. Fernandez, "Image segmentation using Atanassov's intuitionistic fuzzy sets," Expert Systems with Applications, vol. 40, no. 1, pp. 15-26, 2013.

[18] T. Chaira, "Intuitionistic fuzzy segmentation of medical images," IEEE Trans. on Biomedical Engineering, vol. 57, no. 6, pp. 1430-1436, 2010.

[19] S. Kumar and S. S. Gangwar, "Intuitionistic fuzzy time series: an approach for handling non-determinism in time series forecasting," IEEE Trans. on Fuzzy Systems, vol. 24, no. 6, pp. 1270-1281, 2016.

[20] B. P. Joshi and S. Kumar, "A computational method of forecasting based on intuitionistic fuzzy sets and fuzzy time series," in Proc. of the Int'l. Conf. on Soft Computing for Problem Solving, Springer, 2012, pp. 9931000, Dec. 20-22, 2011.

[21] S. S. Gautam, et al., "A refined method of forecasting based on highorder intuitionistic fuzzy time series data," Progress in Artificial Intelligence, vol. 7, no. 4, pp. 339-350, 2018.

[22] I. Eyoh, R. John and G. De Maere, "Interval type-2 a-intuitionistic fuzzy logic for regression problems," IEEE Trans. on Fuzzy Systems, vol. 26, no. 4, pp. 2396-2408, 2018.

[23] I. Eyoh, R. John, G. D. Maere and E. Kayacan, "Hybrid learning for interval type-2 intuitionistic fuzzy logic systems as applied to identification and prediction problems," IEEE Trans. on Fuzzy Systems, vol. 26, no. 5, pp. 2672-2685, 2018.

[24] H. Bustince, et al., "A historical account of types of fuzzy sets and their relationships," IEEE Trans. on Fuzzy Systems, vol. 24, no. 1, pp. 179194, 2016.

[25] G. S. Mahapatra and T. K. Roy, "Intuitionistic fuzzy number and its arithmetic operation with application on system failure," $J$. of Uncertain Systems, vol. 7, no. 2, pp. 92-107, 2013.

[26] J.-S. R. Jang, "ANFIS: Adaptive-network-based fuzzy inference system," IEEE Trans. on Systems, Man, and Cybernetics, vol. 23, pp. 665-684, 1993.

[27] J. M. Mendel, Introduction to Rule-Based Fuzzy Systems, $2^{\text {nd }}$ ed., Springer, Cham, Switzerland, 2017.

[28] P. Angelov, "Crispification: defuzzification over intuitionistic fuzzy sets," Bulletin for Studies and Exchanges on Fuzziness and its Applications, vol. 64, pp. 51-55, 1995.

[29] I. Eyoh, R. John and G. De Maere, Extended Kalman filter-based learning of interval type-2 intuitionistic fuzzy logic system," in Proc. of 2017 IEEE Int'l. Conf. on Systems, Man, and Cybernetics, Banff, Canada, Oct. 2017, pp. 728-733.

[30] I. Eyoh, R. John and G. De Maere, "Interval type-2 intuitionistic fuzzy logic: a comparative evaluation, Proc. of Int'l. Conf. on Information Management of Uncertainty in Knowledge-Based Systems, Springer, Cham, 2018.

[31] D. Wu and J. M. Mendel, "Patch learning," IEEE Trans. on Fuzzy Systems, Early Access, July 2019.

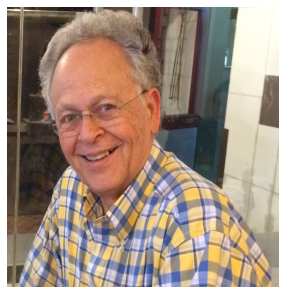

Jerry M. Mendel (LF'04) received the $\mathrm{Ph} . \mathrm{D}$. degree in electrical engineering from the Polytechnic Institute of Brooklyn, Brooklyn, NY.

$\mathrm{He}$ is currently Emeritus Professor of Electrical Engineering at the University of Southern California in Los Angeles, where he has been since

1974. He has published more than 580 technical papers and is author and/or co-author of 13 books, including Uncertain Rule-based Fuzzy Systems: Introduction and New Directions, $2^{\text {nd }} \mathrm{ed}$. His present research interests include: type-2 fuzzy logic systems and computing with words.

Dr. Mendel is a Distinguished Member of the IEEE Control Systems Society and a Fellow of the International Fuzzy Systems Association. Among his awards are the 1983 Best Transactions Paper Award of the IEEE Geoscience and Remote Sensing Society, the 1992 Signal Processing Society Paper Award, the 2002 and 2014 Transactions on Fuzzy Systems Outstanding Paper Awards, a 1984 IEEE Centennial Medal, an IEEE Third Millenium Medal, and a Fuzzy Systems Pioneer Award (2008) from the IEEE Computational Intelligence Society for "fundamental theoretical contributions and seminal results in fuzzy systems".

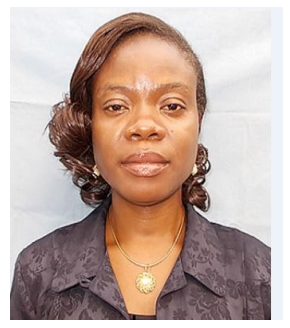

Imo Eyoh (S'16, M'19) received the B.S. and M.S. degrees in Computer Science from the University of Uyo and Ibadan, all in Nigeria, in 1999 and 2005, respectively. She obtained her Ph.D. degree in Computer Science from the University of Nottingham, United Kingdom in 2018. She is currently a lecturer in the Department of Computer Science, University of Uyo. Her main research interest is uncertainty modeling using fuzzy logic. In particular, she works in the area of type-2 fuzzy logic (classical and intuitionistic) with applications in diverse problem domains. She has published many papers in national and international journals and conferences, and is a member of Computer Professionals Registration Council of Nigeria (CPN) and Organization for Women in Science for the Developing World (OWSD).

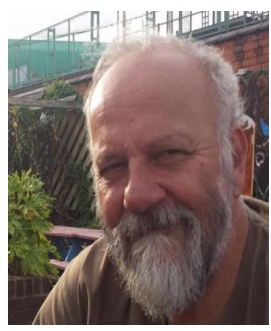

Robert John (M'01, SM'08) received his degree in Mathematics from Leicester Polytechnic in 1979, an MS in Statistics from UMIST in 1981 and a $\mathrm{PhD}$ in type-2 fuzzy logic from De Montfort University in 2000. He is currently Professor of Operational Research and Computer Science and Head of the Optimization and Learning Lab (OPAL) in the School of Computer Science at the University of Nottingham. He is a Fellow of the British Computer Society and elected member of the EPSRC College in the UK. His research interests are primarily around the role of type- 2 fuzzy logic in decision-making. 